\title{
Concomitant hypermethylation of multiple genes in non-small cell lung cancer (NSCLC)
}

\author{
Meiju Ji', Haixia Guan², Bingyin Shi ${ }^{1}$, Peng Hou ${ }^{1}$ \\ ${ }^{1}$ Department of Endocrinology, The First Affiliated Hospital of Xi'an Jiaotong University College \\ of Medicine, Xi'an 710061, the People's Republic of China \\ ${ }^{2}$ Department of Endocrinology and Metabolism, The First Affiliated Hospital of China Medical \\ University, Shenyang 110001, the People's Republic of China
}

\begin{abstract}
Primary lung cancer remains the leading cause of cancer death worldwide. Promoter hypermethylation is a major inactivation mechanism of tumor-related genes, and increasingly appears to play an important role in carcinogenesis. In the present study, we used quantitative methylation-specific PCR (Q-MSP) assays to analyze promoter hypermethylation of nine genes in a large cohort of well-characterized non-small cell lung cancer (NSCLC) and explore their associations with the clinicopathological features of tumor. We found that there were significant differences in methylation levels for six of nine gene promoters between cancerous and noncancerous lung tissues. More importantly, with $100 \%$ diagnostic specificity, high sensitivity, ranging from $44.9 \%$ to $84.1 \%$, was found for each of the nine genes. Interestingly, promoter hypermethylation of most genes was closely associated with histologic type, which was more frequent in squamous cell carcinomas (SCC) than in adenocarcinomas (ADC). In addition, highly frequent concomitant methylation of multiple genes was found in NSCLC, particularly in SCC. Our data showed that multiple genes were aberrantly methylated in lung tumorigenesis, and that they were closely associated with certain clinicopathological features of NSCLC, particularly of the histologic type, suggesting that these hypermethylated genes could be potential biomarkers in early detection of NSCLC in high-risk individuals, as well as in evaluating the prognosis of NSCLC patients. (Folia Histochemica et Cytobiologica 2011, Vol. 49, No. 1, 132-141)
\end{abstract}

Key words: DNA methylation, multiple genes, quantitative methylation-specific PCR (Q-MSP), non-small cell lung cancer (NSCLC)

\section{Introduction}

Worldwide, lung cancer kills more than one million people each year, and as the leading cause of death in men, and the second leading cause in women, it is a major health problem $[1,2]$. Lung cancer is clinically classified into two major histological categories: small cell lung cancer (SCLC) and non-small cell lung cancer (NSCLC). The former is the most aggressive subtype, accounting for $10-15 \%$ of all cases. NCLSC

Correspondence address: P. Hou, Department of Endocrinology, the First Affiliated Hospital of Xi'an Jiaotong University College of Medicine, Xi'an 710061, the People's Republic of China; e-mail:phou@mail.xjtu.edu.cn accounts for approximately 85-90\% of lung cancers [2]. Despite surgery, approximately $30-40 \%$ of patients with NSCLC who have discrete lesions and histologically negative lymph nodes die of recurrent disease [3]. One reason for this problem is that this disease has proven difficult to diagnose early and treat successfully. In fact, many imaging and cytology-based strategies have been employed to achieve early detection of lung cancer and identified high-risk individuals. Although some of these strategies are indeed sensitive, none has been demonstrated to reduce lung cancer mortality. Fortunately, specific molecular markers would provide an alternative approach to complement imaging techniques for the early diagnosis of lung cancer. Among them, DNA methylation has emerged as a highly promising biomarker, and it is being actively studied in multiple cancers. 
Hypermethylation of gene promoter is an important mechanism of gene inactivation and acts as an alternative to mutational events, such as gene mutations and intragenic deletions, in oncogenesis $[4,5]$. Hypermethylation of gene promoter is the earliest and commonest molecular event in human cancers, including NSCLC [6, 7], indicating that loss of gene transcription mediated by methylation events is recognized now as a crucial component in the initiation and progression of lung cancer. Taken together, hypermethylation of gene promoter may be an important biomarker for the early detection of lung cancer.

In the present study, we determined the DNA methylation profiles of nine genes using quantitative methylation-specific PCR (Q-MSP) in a cohort of clinically well-characterized NSCLC samples. The selected genes play important roles in T- and B-cell regulation in certain malignancies (CALCA), cell adhesion and/or invasion and/or metastasis (CDH1), apoptosis (DAPK1), development (EVX2 and IRX2), cell proliferation and/or differentiation (PAX6, $\mathrm{RAR} \beta$, and TIMP3), and cell cycle control (RASSF1A).

The aim of this study was to identify a panel of genes that differentiate cancerous tissues from noncancerous tissues as excellent biomarkers for early detection and prognostic evaluation of lung cancer, and explore their associations with known or suspected cancer risk factors.

\section{Material and methods}

\section{Clinical samples}

With institutional approval, a series of 96 paraffin-embedded primary NSCLC samples and 15 noncancerous lung samples were randomly selected at the First Affiliated Hospital of China Medical University, People's Republic of China. None of these patients had received chemotherapy or radiotherapy before the surgery. Informed consent was obtained from each patient before the surgery. The histopathological diagnosis and subtype classification of NSCLC samples were examined and agreed upon by an experienced pathologist at the Department of Pathology of the Hospital. As shown in Table 1 , the mean age of all NSCLC cases was 58.9 years. Those aged $>60$ years accounted for $42 \%$. There were more males than females (69\% vs. $31 \%)$. Sixty-nine percent of patients had a smoking history. Ninety per cent of NSCLC cases had surgical stage I and II disease and $84 \%$ had tumors $<5 \mathrm{~cm}$. By histology, $69 \%$ were squamous cell carcinomas (SCC), the remaining $31 \%$ being adenocarcinomas (ADC). Cases with lymph node metastasis, pleural indentation and invasion or adhesion were 26/96 (27\%), 21/96 (22\%), and 31/96 $(32 \%)$, respectively.
Table 1. Baseline patient and tumor characteristics of NSCLC cases

\begin{tabular}{|c|c|}
\hline Characteristics & No. of patients (\%) \\
\hline \multicolumn{2}{|l|}{ Gender } \\
\hline Male & $66(68.8)$ \\
\hline Female & $30(31.2)$ \\
\hline Age (mean years $\pm \mathrm{SD})$ & $58.9 \pm 9.2$ \\
\hline$\leq 60$ & $56(58.3)$ \\
\hline$>60$ & $40(41.7)$ \\
\hline \multicolumn{2}{|l|}{ Smoking history (pack-years) } \\
\hline 0 & $30(31.3)$ \\
\hline $1-39$ & $35(36.5)$ \\
\hline$\geq 40$ & $31(32.2)$ \\
\hline Tumor size (mean $\mathrm{cm} \pm \mathrm{SD})$ & $3.9 \pm 1.7$ \\
\hline $1-3$ & $37(38.5)$ \\
\hline $3-5$ & $44(45.8)$ \\
\hline$>5$ & $15(15.7)$ \\
\hline \multicolumn{2}{|l|}{ Histologic type } \\
\hline Adenocarcinoma & $30(31.3)$ \\
\hline Bronchoalveolar cell (BAC) & $6(6.3)$ \\
\hline Adenocarcinoma (non-BAC) & $24(25)$ \\
\hline Squamous & $66(68.7)$ \\
\hline \multicolumn{2}{|l|}{ Histologic stage } \\
\hline I & $54(56.3)$ \\
\hline II & $32(33.3)$ \\
\hline III & $10(10.4)$ \\
\hline \multicolumn{2}{|l|}{ Lymph node metastasis } \\
\hline No & $70(72.9)$ \\
\hline Yes & $26(27.1)$ \\
\hline \multicolumn{2}{|l|}{ Pleural indentation } \\
\hline No & $75(78.1)$ \\
\hline Yes & $21(21.9)$ \\
\hline \multicolumn{2}{|l|}{ Invasion or adhesion } \\
\hline No & $65(67.7)$ \\
\hline Yes & $31(32.3)$ \\
\hline
\end{tabular}

\section{DNA isolation}

Paraffin-embedded NSCLC samples were treated and DNA was isolated as previously described [8]. Briefly, after an eight-hour treatment at room temperature with xylene to remove paraffin, samples were incubated with $1 \%$ sodium dodecyl sulfate (SDS) and $0.5 \mathrm{mg} / \mathrm{ml}$ proteinase $\mathrm{K}$ at $48^{\circ} \mathrm{C}$ for 48 hours to digest tissues. A few mid-interval spiking aliquots of concentrated proteinase $\mathrm{K}$ facilitated digestion. DNA was subsequently isolated by standard phenol-chloroform extraction and ethanol precipitation. 


\section{Sodium bisulfite treatment}

Genomic DNA from the primary tumors and noncancerous lung samples was subjected to treatment of sodium bisulfite, as described previously [9]. Briefly, a final volume of $20 \mu \mathrm{l}$ of $\mathrm{H}_{2} \mathrm{O}$ containing $\sim 2 \mu \mathrm{g}$ genomic DNA, $10 \mu \mathrm{g}$ salmon sperm DNA, and $0.3 \mathrm{M}$ $\mathrm{NaOH}$ was incubated at $50^{\circ} \mathrm{C}$ for 20 minutes to denature the DNA. The mixture was then incubated for $2-3$ hours at $70^{\circ} \mathrm{C}$ in $500 \mu 1$ of a freshly prepared solution containing $3 \mathrm{M}$ sodium bisulfite (Sigma, St. Louis, MO, USA), and $10 \mathrm{mM}$ hydroquinone (Sigma, St. Louis, MO, USA). DNA was subsequently recovered by a Wizard DNA Clean-Up System (Promega Corp., Madison, WI, USA) following the instructions of the manufacturer, followed by ethanol precipitation, and re-suspension in $30 \mu 1$ of deionized $\mathrm{H}_{2} \mathrm{O}$. After bisulfite treatment, all unmethylated cytosine residues converted to uracil, whereas the methylated cytosine residues remained unchanged. Bisulfite-treated DNA samples were stored at $-80^{\circ} \mathrm{C}$ until use.

\section{Quantitative methylation-specific PCR (Q-MSP) assay}

The methylation status was determined by the fluorescence-based quantitative PCR assay as described previously [9]. Briefly, the Q-MSP assay was carried out in triplicate for each sample in a final reaction mixture of $20 \mu \mathrm{l}$ containing $3 \mu \mathrm{l}$ bisulfitetreated DNA, $600 \mathrm{nM}$ each primer, $200 \mathrm{nM}$ Taq-Man probe, $5.5 \mathrm{mM} \mathrm{MgCl} 2,1 \mathrm{U}$ platinum Taq polymerase, $200 \mu \mathrm{M}$ each of deoxyguanosine triphosphate, and $2 \%$ Rox reference. After an initial denaturation step at $95^{\circ} \mathrm{C}$ for two minutes, 40 cycles of 15 seconds at $95^{\circ} \mathrm{C}$ and one minute at $60^{\circ} \mathrm{C}$ for annealing and extension were run using a CFX96 Real-Time PCR Detection System (Bio-Rad, CA, USA). Normal leukocyte DNA was methylated in vitro with Sss I methylase (New England Biolabs, Beverly, MA, USA) to generate completely methylated DNA as a positive control. Each plate contained triplicate samples, multiple water blanks, as well as negative unmethylated controls and serial dilutions of positive methylated control to construct the standard curve. The internal reference gene $\beta$-actin was used to normalize the amount of input DNA. The primers and TaqMan probes used in the present study were designed using Methyl Primer Express Software v1.0 (Applied Biosystems) and presented in Table 2. The relative methylation level of each sample was calculated using the method described previously [9].

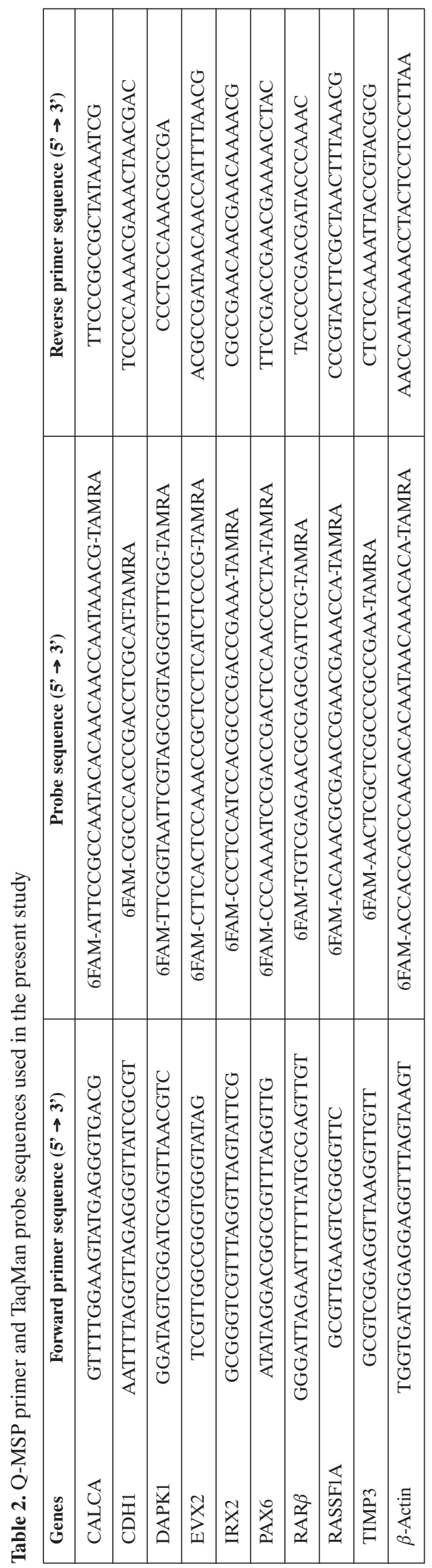




\section{Data analysis}

A positive gene methylation was defined when the ratio was above a certain cut-off value. The relative methylation level varied substantially among the nine genes and the cut-off points were thus studied for each gene individually. To determine cut-off value of each gene, we construct receiver operating characteristic (ROC) curves using MedCalc software (MedCalc, Belgium). The area under the ROC curve is a measure of the ability of a continuous marker to accurately classify cancerous and noncancerous tissue. Such a curve is a plot of sensitivity vs. 100 minus specificity values associated with all dichotomous markers that can be formed by varying the cut-off values used to define a marker 'positive gene methylation'. Logistic regression models were used to examine the association between gene methylation and the clinicopathological features of cases using the MedCalc software (MedCalc, Belgium). First, univariate models were examined, then multivariate models were developed that adjusted for the most important covariates, including age, pack-years, histologic type, and histologic stage.

Associations were expressed as odds ratios and their corresponding 95\% confidence intervals (CI). Analyses were conducted to assess the association with NSCLC for each gene separately, then for concomitant hypermethylation of multiple genes. $p<0.05$ was considered to be statistically significant. All statistical analyses were performed using the SPSS statistical package v11.5 (Chicago, IL, USA).

\section{Results}

\section{Frequent promoter hypermethylation in NSCLC}

The methylation profiles of nine genes were assessed in their promoters using Q-MSP in a cohort of NSCLC samples, including CALCA, CDH1, DAPK1, EVX2, IRX2, PAX6, RAR $\beta$, RASSF1A, and TIMP3. As shown in Figure 1, the overall methylation level of each of the nine genes was higher in cancerous tissues than in noncancerous lung tissues. Among them, significant differences were found in CALCA $(\mathrm{p}<$ $<0.01)$, CDH1 ( $<<0.01)$, EVX2 ( $<<0.01)$, PAX6 $(\mathrm{p}<0.01), \operatorname{RAR} \beta(\mathrm{p}<0.01)$, and RASSF1A $(\mathrm{p}<0.05)$. The cut-off values for each gene were calculated using ROC curves to differentiate NSCLC from noncancerous lung tissues, respectively, and determine diagnostic sensitivity and specificity. As shown in Figure 2 , with more weight toward specificity than sensitivity, $100 \%$ diagnostic specificity was obtained for all of these genes. The sensitivity of each individual gene was $69.2 \%$ for CALCA, $45.8 \%$ for CDH1, $84.1 \%$ for DAPK1, $63.6 \%$ for EVX2, 52.3\% for IRX2, 75.7\% for PAX6, $54.2 \%$ for RAR $\beta, 72.9 \%$ for RASSF1A, and $44.9 \%$ for TIMP3.

\section{Association of promoter hypermethylation with clinicopathologic features of NSCLC}

Among all clinicopathologic features, methylation levels varied substantially by histologic type (Table 3 ). Methylation level of most genes was higher in SCC than in ADC, including CALCA, CDH1, DAPK1, EVX2, IRX2, RAR $\beta$, and TIMP3, particularly in DAPK1 ( $<0.01)$ and TIMP3 (p < 0.01) (Table 3). Conversely, methylation level of RASSF1A was much higher in ADC than in SCC (Table 3), although no significant difference was observed $(p=0.08)$. There was no significant association between gene methylation and age (Table 3). Among all hypermethylated genes, methylation level of CALCA was only found to be significantly associated with smoking history $(p<0.05)$ (Table 3). Methylation level of all of these genes was not significantly associated with invasion and adhesion (Table 3 ). Table 3 also shows that there was a significantly lower methylation level of RASSF1A in the lymph node metastasis group than in the nonmetastasis group ( $\mathrm{p}<0.05)$. Moreover, methylation level of CALCA was found to be significantly associated with histologic stage $(p<0.05)$ (Table 3). However, no association was found between promoter hypermethylation and other clinocopathologic features, including gender, quantity of cigarette smoking (pack-years), tumor size, or pleural indentation (data not shown).

In univariate analyses, hypermethylation of most genes was more frequent in SCC compared to ADC, particularly in CDH1 $(\mathrm{OR}=2.63,95 \% \mathrm{CI}=1.05-$ -6.60), DAPK1 (OR $=6.64,95 \% \mathrm{CI}=1.85-23.8)$, IRX2 $(\mathrm{OR}=2.89,95 \% \mathrm{CI}=1.17-7.13)$, and TIMP3 $(\mathrm{OR}=2.51,95 \% \mathrm{CI}=1.01-6.21)($ Table 4$)$. Conversely, hypermethylation of RASSF1A was significantly frequent in $\mathrm{ADC}(\mathrm{OR}=0.31,95 \% \mathrm{CI}=0.09$ -0.99 ) in the present study (Table 4). In addition, hypermethylation of CALCA $(\mathrm{OR}=2.13,95 \% \mathrm{CI}=$ $=1.01-4.47)$ and TIMP3 (OR $=1.88,95 \% \mathrm{CI}=1.01-$ -3.49) was significantly associated with histologic stage (Table 4). Of note, hypermethylation of $\operatorname{RAR} \beta$ was negatively associated with smoking history $(\mathrm{OR}=$ $=0.50,95 \% \mathrm{CI}=0.20-1.23)$, quantity of cigarette smoking $(\mathrm{OR}=0.60,95 \% \mathrm{CI}=0.36-1.01)$ and pleural indentation $(\mathrm{OR}=0.41,95 \% \mathrm{CI}=0.15-1.11)$ in NSCLC, although no statistical significance was noted (Table 4). Additionally, hypermethylation of RASSF1A (OR $=0.47,95 \%$ CI $=0.24-0.90)$ was significantly negatively associated with histologic stage 
CALCA
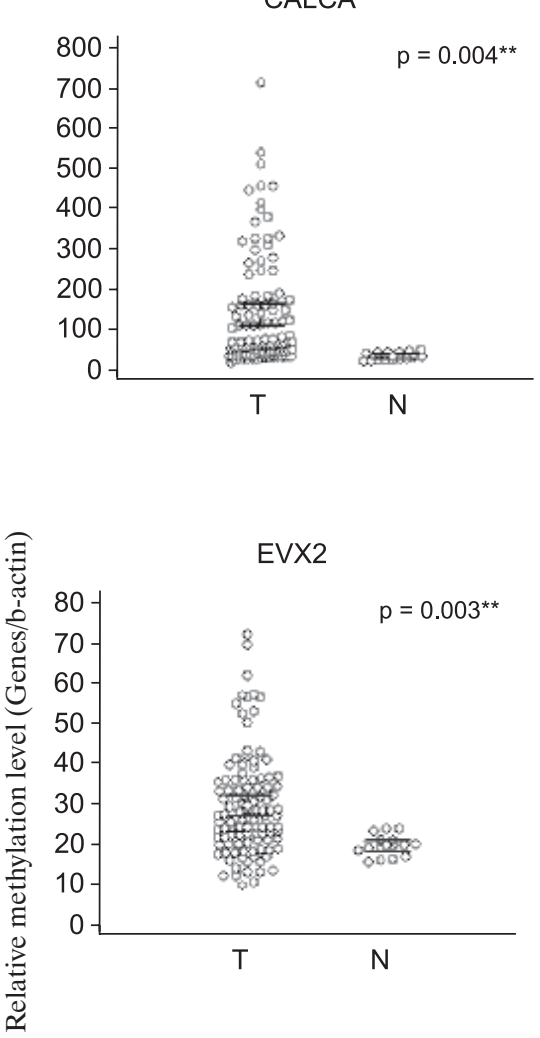

$\operatorname{RAR} \beta$

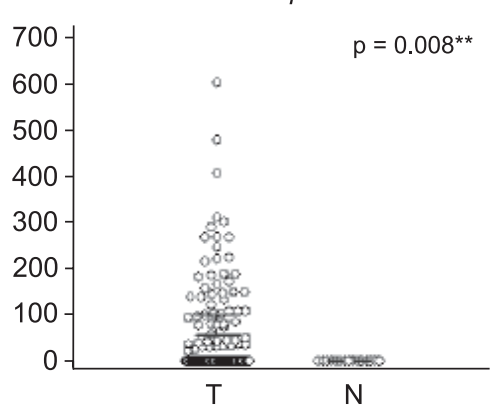

$\mathrm{CDH} 1$

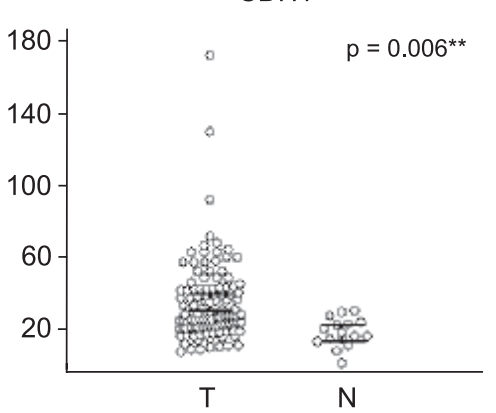

IRX2

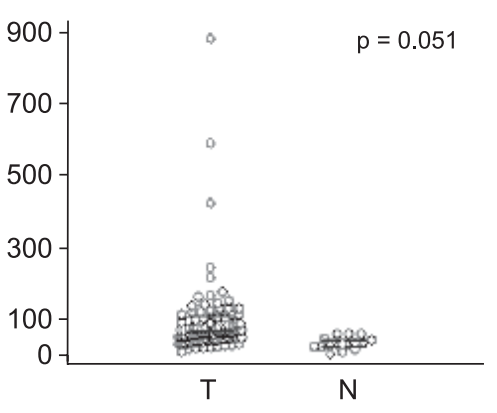

RASSF1A

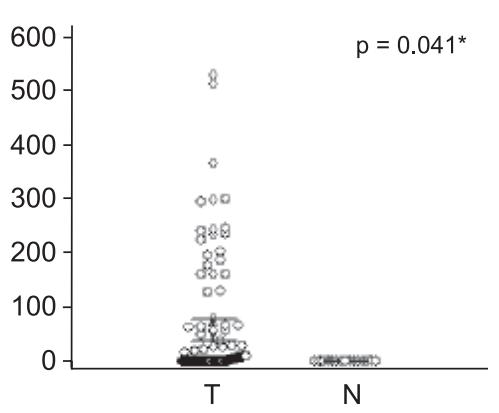

DAPK1
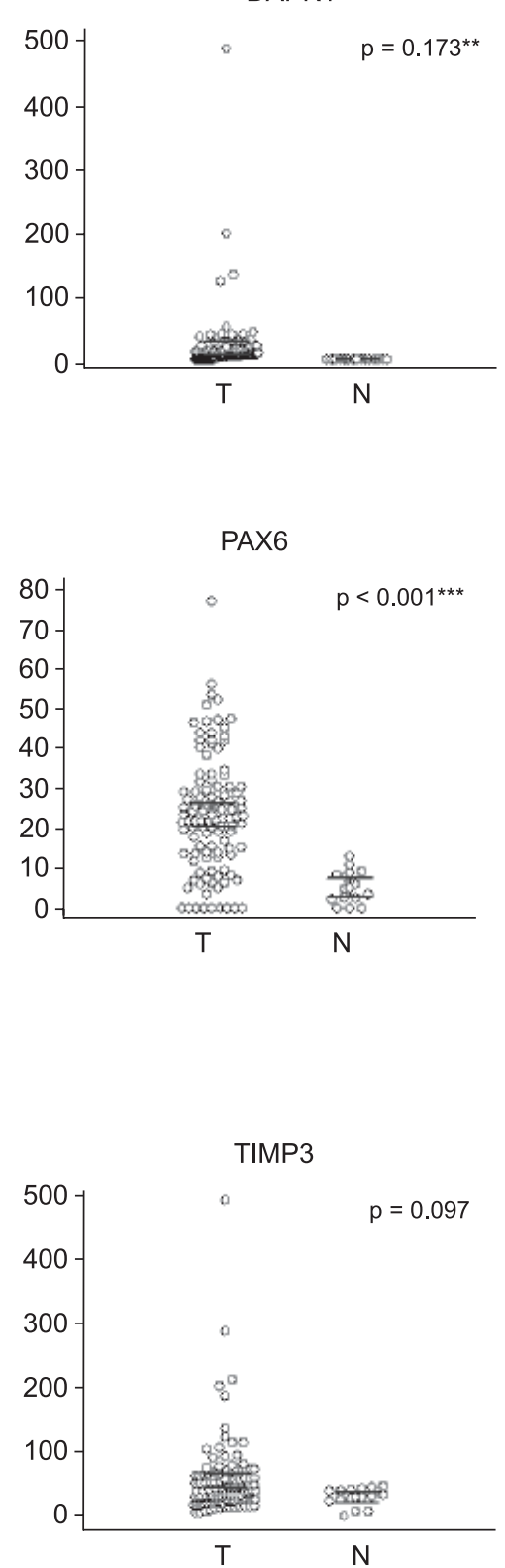

Figure 1. Distribution patterns of promoter hypermethylation of the nine genes in NSCLC. Quantitative methylation-specific PCR (Q-MSP) assay was performed as described in Material and methods. The relative methylation level (on Y axis) is represented by ratios of candidate gene $/ \beta$-actin $\times 1,000$, except for EVX2 and PAX6/ $\beta$-actin $\times 100$. Horizontal lines indicate a $95 \%$ normal confidence interval for the sample mean. $\mathrm{T}$ - cancerous tissues; $\mathrm{N}$ - noncancerous lung tissues. ${ }^{*} \mathrm{p}<0.05 ; * \mathrm{p}<0.01 ; * * \mathrm{p}<0.001$

(Table 4). Similarly, hypermethylation of RASSF1A was also negatively associated with smoking history $(\mathrm{OR}=0.58,95 \% \mathrm{CI}=0.20-1.62)$, lymph node metastasis $(\mathrm{OR}=0.47,95 \% \mathrm{CI}=0.18-1.25)$, and invasion or adhesion $(\mathrm{OR}=0.44,95 \% \mathrm{CI}=0.17-1.11)$, respectively, although these associations did not reach statistical difference (Table 4). In order to assess the independent associations between gene methylation and age, pack-years, histologic type, and histologic stage, we conducted multiple multivariable logistic regressions (Table 5).
In multivariable analyses adjusting for the potential false discovery rate associated with multiple comparisons (nine different models), hypermethylation of $\mathrm{CDH} 1(\mathrm{OR}=3.14,95 \% \mathrm{CI}=1.14-8.65)$, DAPK1 $(\mathrm{OR}=8.07,95 \% \mathrm{CI}=1.75-37.2)$, and IRX2 (OR $=3.54,95 \% \mathrm{CI}=1.29-9.73)$ remained associated with histologic type, which was significantly more likely in SCC than in ADC (Table 5). However, after adjustment, there were no significant differences between hypermethylation of RASSF1A and TIMP3 and histologic type (Table 5). Interestingly, 


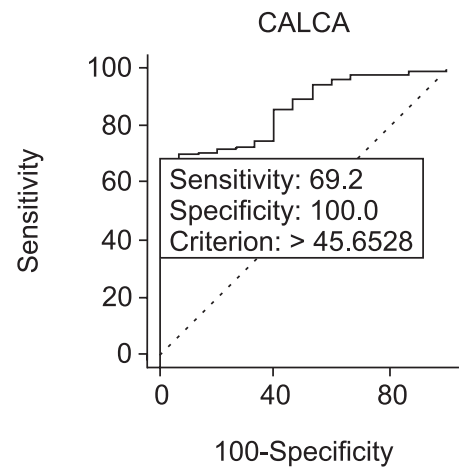

EVX2

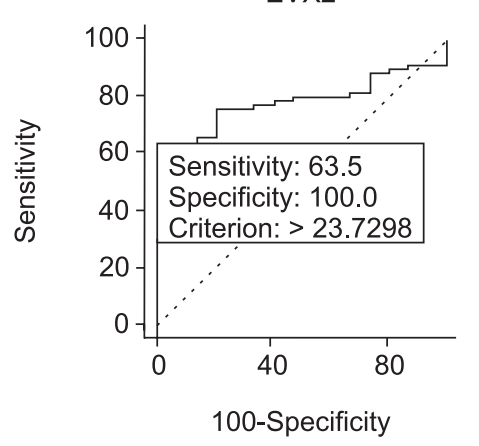

RAR b

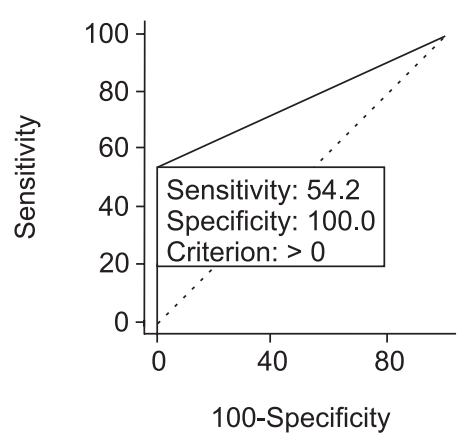

$\mathrm{CDH} 1$

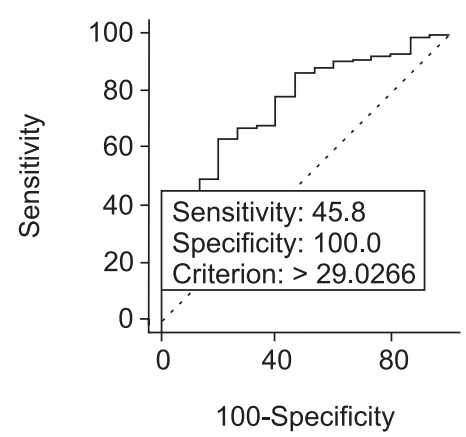

IRX2

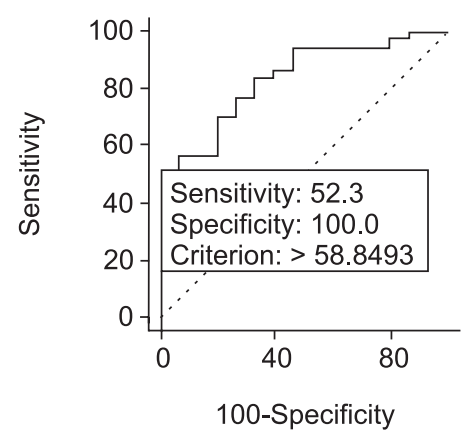

RASSF1A

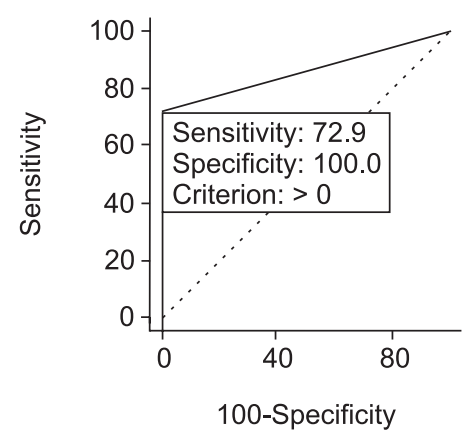

DAPK1

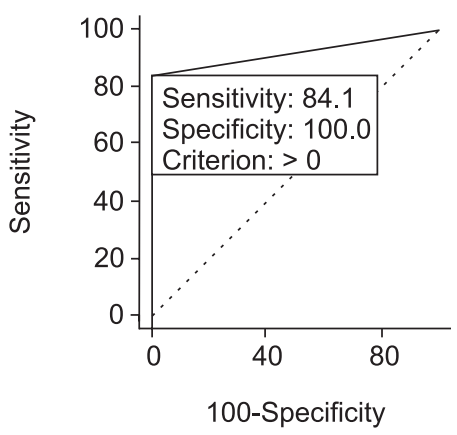

PAX6

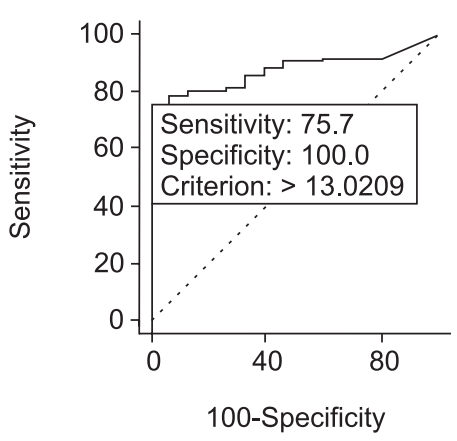

TIMP3

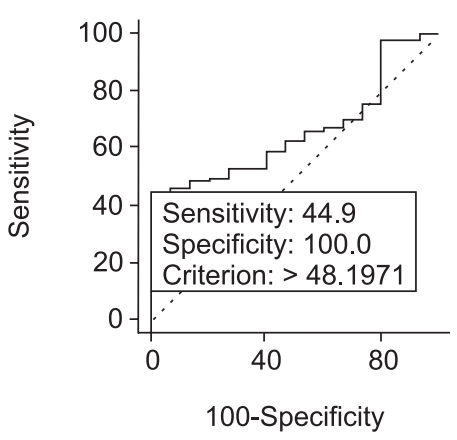

Figure 2. Receiver operating characteristic (ROC) curves for the nine genes in NSCLC. Complete DNA methylation data from all cancerous and noncancerous lung tissues were used for the analyses. The ROC curves plot sensitivity vs. 100\%-specificity. The cut-off values for each of nine genes were determined based on ROC curves individually

hypermethylation of $\operatorname{RAR} \beta$ remained negatively associated with quantity of cigarette smoking after multivariable adjustment, and a statistical difference was noted $(\mathrm{OR}=0.58,95 \% \mathrm{CI}=0.34-0.99)$ (Table 5). Moreover, multivariable analyses indicated that no significant differences were found between hypermethylation of CALCA and TIMP3 and histologic stage (Table 5). However, hypermethylation of RASSF1A (OR $=0.55,95 \%$ CI $=0.27-1.10)$ remained negatively associated with histologic type (Table 5), although this association did not reach statistical difference.

\section{Concomitant hypermethylation of multiple genes and their associations with clinocopathologic features in NSCLC}

Field cancerization effects in NSCLC will probably dictate the development of a gene panel for early detection of NSCLC that focuses on assessing the prevalence for promoter hypermethylation of multiple genes in individual specimens. In the present study, we identified a panel of the nine hypermethylated genes with $100 \%$ diagnostic specificity in NSCLC, and further analyzed methylation status of these genes in indivi- 


\begin{tabular}{|c|c|c|c|c|c|c|c|c|c|c|c|c|c|c|c|c|c|c|c|c|c|c|c|}
\hline \multirow{4}{*}{ 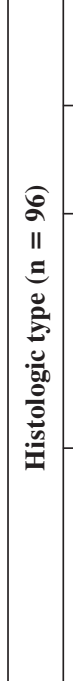 } & \multicolumn{2}{|l|}{$a$} & $\begin{array}{l}\tilde{b} \\
0 \\
0\end{array}$ & $\stackrel{\overbrace{}}{\varrho}$ & 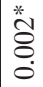 & 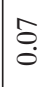 & 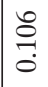 & 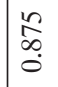 & 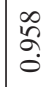 & $\stackrel{\infty}{\stackrel{\infty}{0}}$ & 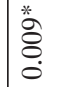 & \multirow{4}{*}{ 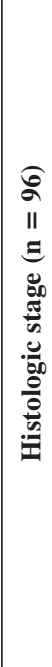 } & \multicolumn{2}{|l|}{$\theta$} & $\begin{array}{l}\stackrel{*}{*} \\
\stackrel{\delta}{0} \\
\stackrel{0}{0}\end{array}$ & $\begin{array}{l}\text { ơ } \\
\stackrel{0}{0}\end{array}$ & $\mid$\begin{tabular}{c}
$\mathscr{0}$ \\
\multirow{3}{0}{} \\
0
\end{tabular} & $\begin{array}{c}3 \\
\tilde{s} \\
\tilde{s}\end{array}$ & O̊̊ & 年 & 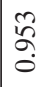 & 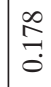 & $\underset{⿱}{\stackrel{t}{0}}$ \\
\hline & $\mathbf{N}$ & & & $\stackrel{g}{\vec{f}}$ & 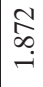 & ָे & 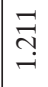 & 合 & $\begin{array}{l}\hat{\sigma} \\
\hat{\sigma} \\
0\end{array}$ & 웜 & 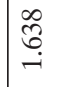 & & \multicolumn{2}{|l|}{$\mathbf{N}$} & $\begin{array}{l}\stackrel{\text { I }}{\rightarrow} \\
.\end{array}$ & $\stackrel{\infty}{\stackrel{\overbrace{}}{\circ}}$ & $\mid \begin{array}{l}\infty \\
\stackrel{0}{0} \\
\stackrel{0}{0}\end{array}$ & 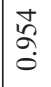 & $\begin{array}{l}\overrightarrow{5} \\
\text { n. }\end{array}$ & 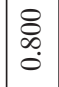 & $\frac{n}{n}$ & $\stackrel{\overbrace{}}{\rightleftarrows}$ & 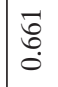 \\
\hline & 岁 & $\begin{array}{l}\widetilde{ల} \\
\text { II } \\
\Xi\end{array}$ & 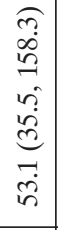 & 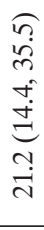 & 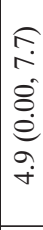 & 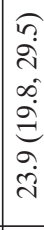 & 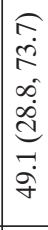 & 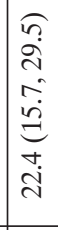 & 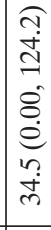 & 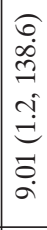 & 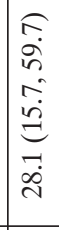 & & $\underset{v}{\Xi}$ & $\begin{array}{l}\widehat{\infty} \\
\text { II } \\
\Xi\end{array}$ & 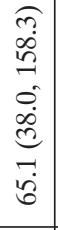 & 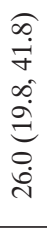 & 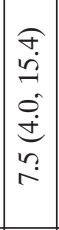 & 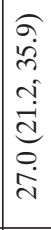 & 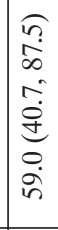 & 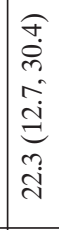 & 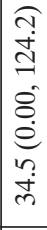 & 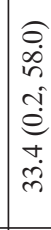 & $\begin{array}{l}\hat{\sigma} \\
\dot{i} \\
\hat{d} \\
d \\
d \\
\dot{d} \\
\dot{\sigma}\end{array}$ \\
\hline & D & $\begin{array}{l}8 \\
\text { II } \\
\Xi\end{array}$ & 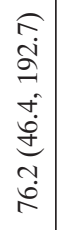 & 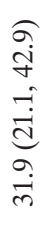 & 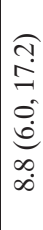 & 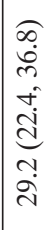 & 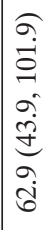 & 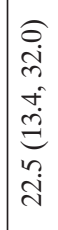 & 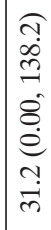 & 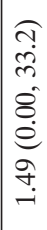 & 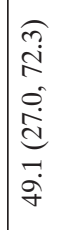 & & $\underset{\Lambda}{\Xi}$ & $\begin{array}{l}\Theta \\
\Theta \\
\| \\
\Xi\end{array}$ & 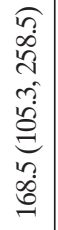 & 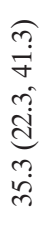 & 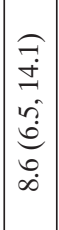 & 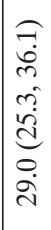 & 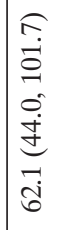 & 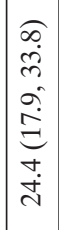 & $\begin{array}{l}0 \\
\dot{0} \\
\dot{0} \\
0 \\
0 \\
8 \\
e \\
0 \\
0 \\
\dot{I}\end{array}$ & 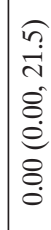 & 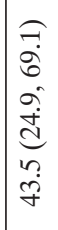 \\
\hline \multirow{4}{*}{ 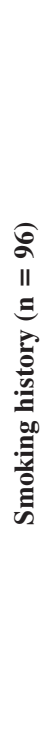 } & \multicolumn{2}{|l|}{$a$} & $\begin{array}{l}\stackrel{*}{*} \\
\stackrel{\Delta}{0} \\
\stackrel{0}{0}\end{array}$ & $\begin{array}{l}\mathscr{0} \\
\stackrel{0}{0} \\
\stackrel{0}{0}\end{array}$ & $\begin{array}{l}2 \\
\hat{n} \\
n \\
o\end{array}$ & م̂ & $\stackrel{\vec{\jmath}}{\stackrel{0}{0}}$ & 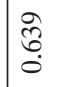 & $\begin{array}{l}\infty \\
\tilde{N} \\
\tilde{c}\end{array}$ & तु & 菏 & \multirow{4}{*}{ 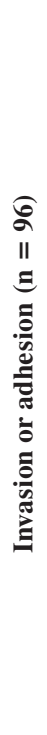 } & \multicolumn{2}{|l|}{$\theta$} & 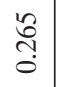 & $\begin{array}{l}\vec{t} \\
\stackrel{d}{0}\end{array}$ & 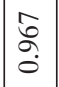 & $\begin{array}{l}R \\
n \\
0\end{array}$ & \&̊. & $\begin{array}{l}\mathscr{L} \\
\infty \\
\infty \\
0 \\
0\end{array}$ & 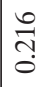 & חֶֶ & $\begin{array}{l}\infty \\
\stackrel{0}{0} \\
0\end{array}$ \\
\hline & \multicolumn{2}{|l|}{$\mathbf{N}$} & 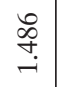 & $\begin{array}{l}\stackrel{0}{\stackrel{0}{c}} \\
\stackrel{0}{0}\end{array}$ & 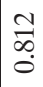 & $\underset{i}{ }$ & $\begin{array}{l}\infty \\
\infty \\
0 \\
0\end{array}$ & $\frac{\mathscr{m}}{\stackrel{f}{\delta}}$ & 号 & $\stackrel{\widetilde{\delta}}{\stackrel{\leftrightarrow}{-}}$ & $\begin{array}{l}\tilde{O} \\
\stackrel{\infty}{0} \\
0\end{array}$ & & \multicolumn{2}{|c|}{$N$} & 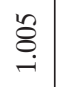 & 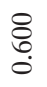 & 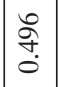 & 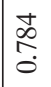 & 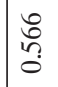 & $\begin{array}{l}\hat{0} \\
0 \\
0\end{array}$ & 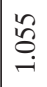 & $\begin{array}{l}\hat{\delta} \\
\infty \\
0 \\
0\end{array}$ & 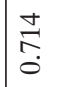 \\
\hline & $z$ & 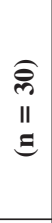 & 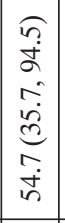 & 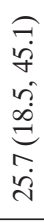 & $\begin{array}{l}\sigma \\
\tilde{d} \\
\sim \\
\sim \\
\tilde{d} \\
\sim \\
\alpha \\
\infty\end{array}$ & 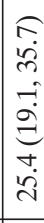 & 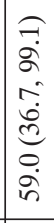 & 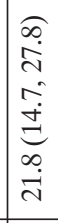 & 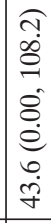 & 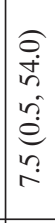 & 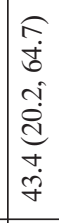 & & z & $\begin{array}{l}68 \\
\| \\
\| \\
\Xi\end{array}$ & 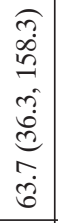 & 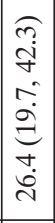 & 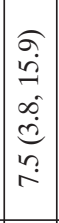 & 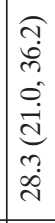 & 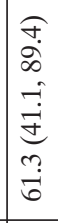 & 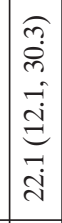 & 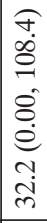 & $\begin{array}{l}\hat{E} \\
\dot{\delta} \\
m \\
\tilde{e} \\
n \\
m \\
m\end{array}$ & 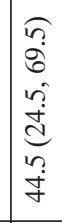 \\
\hline & $\lambda$ & & 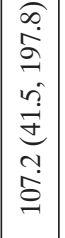 & 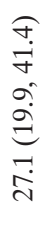 & 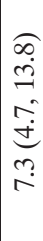 & 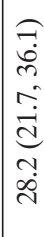 & 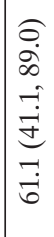 & 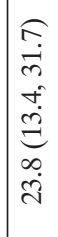 & 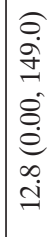 & 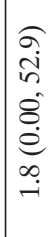 & 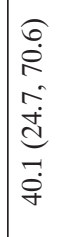 & & $\lambda$ & $\begin{array}{l}\text { ले } \\
\text { ॥I } \\
\text { }\end{array}$ & 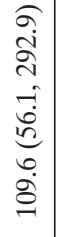 & 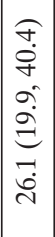 & 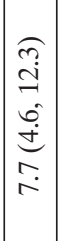 & 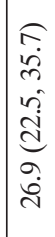 & 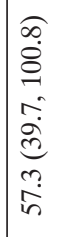 & 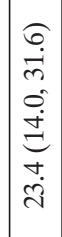 & 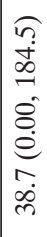 & 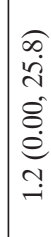 & 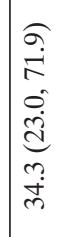 \\
\hline \multirow{4}{*}{ 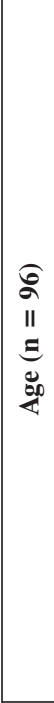 } & $a$ & & $\mid \begin{array}{c}\tilde{N} \\
f \\
\end{array}$ & $\begin{array}{l}\stackrel{R}{0} \\
\stackrel{0}{\circ}\end{array}$ & $\begin{array}{l}\text { oे } \\
\text { ते }\end{array}$ & ণ্ণ & $\underset{0}{\tilde{O}}$ & 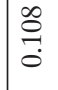 & f & $\mid \begin{array}{l}2 \\
\infty \\
\infty \\
0\end{array}$ & $\begin{array}{l}0 \\
\infty \\
\infty \\
0 \\
0\end{array}$ & \multirow{4}{*}{ 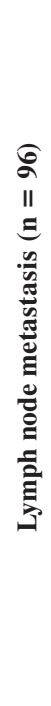 } & \multicolumn{2}{|l|}{ a } & $\stackrel{\hat{\sigma}}{\overrightarrow{0}}$ & 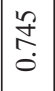 & \begin{tabular}{|l}
$\infty$ \\
0 \\
0 \\
0
\end{tabular} & $\begin{array}{l}\hat{0} \\
\tilde{0} \\
0\end{array}$ & $\begin{array}{c}\underset{1}{0} \\
\infty \\
0 \\
0\end{array}$ & $\underset{\dot{0}}{\stackrel{t}{i}}$ & 点 & 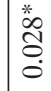 & 吕 \\
\hline & $\mathbf{N}$ & & $\mid \begin{array}{c}n \\
\substack{1 \\
\infty \\
0 \\
0}\end{array}$ & 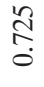 & $\underset{\dot{\sigma}}{\overrightarrow{-}}$ & $\underset{\tilde{\delta}}{\stackrel{\sim}{r}}$ & $\frac{\text { I }}{\stackrel{f}{0}}$ & 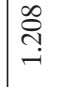 & $\frac{0}{2}$ & $\begin{array}{l}\text { Dे } \\
0 \\
0\end{array}$ & $\mid \begin{array}{l}\infty \\
0 \\
0 \\
0\end{array}$ & & \multicolumn{2}{|l|}{$\mathbf{N}$} & $\underset{\stackrel{S}{0}}{\stackrel{-}{-}}$ & $\begin{array}{l}2 \\
\hat{\sigma} \\
0 \\
0\end{array}$ & $\mid \begin{array}{l}\hat{\mathscr{o}} \\
\stackrel{0}{0}\end{array}$ & $\overrightarrow{\widehat{\sigma}}$ & $\begin{array}{l}\tilde{C} \\
\text { ర్ర } \\
0\end{array}$ & 宊 & 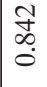 & $\stackrel{\text { sิ }}{\text { f }}$ & $\begin{array}{l}\stackrel{n}{\alpha} \\
\stackrel{\alpha}{0}\end{array}$ \\
\hline & $\begin{array}{l}8 \\
\text { VI }\end{array}$ & & 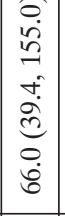 & 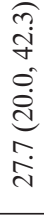 & 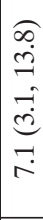 & 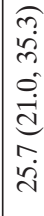 & 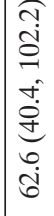 & 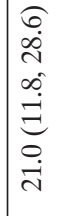 & 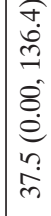 & 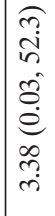 & $\begin{array}{l}\hat{\sigma} \\
\infty \\
0 \\
\infty \\
\infty \\
d \\
d \\
0 \\
0 \\
\dot{m}\end{array}$ & & z & $\begin{array}{l}\widehat{尺} \\
\text { II } \\
\text { }\end{array}$ & 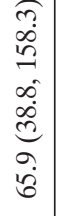 & 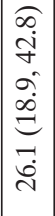 & 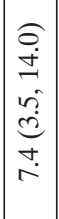 & 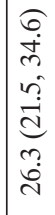 & 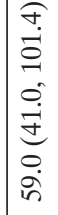 & 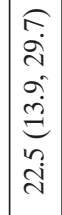 & 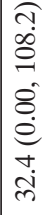 & 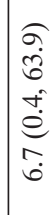 & 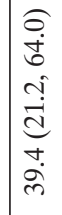 \\
\hline & $\begin{array}{l}8 \\
1\end{array}$ & & 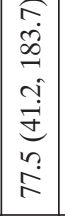 & 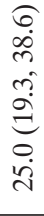 & 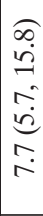 & 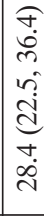 & 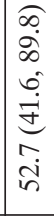 & 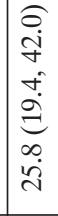 & 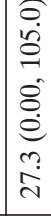 & 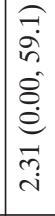 & 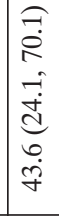 & & 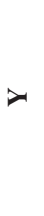 & $\begin{array}{l}\text { ¿ } \\
\text { II } \\
\Xi\end{array}$ & 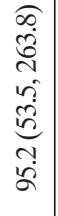 & 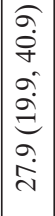 & 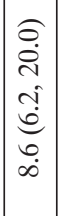 & 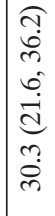 & 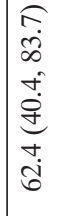 & 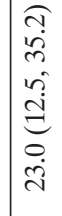 & 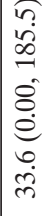 & 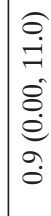 & $\begin{array}{l}\widehat{m} \\
\tilde{i} \\
\tilde{n} \\
\tilde{d} \\
\tilde{d} \\
\vec{i} \\
i n\end{array}$ \\
\hline$\Xi$ & & & $\begin{array}{l}\mathbb{U} \\
\text { 它 } \\
\end{array}$ & $\tilde{\sigma}$ & $\frac{a}{a}$ & 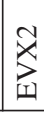 & $\begin{array}{l}\mathfrak{x} \\
\stackrel{\Xi}{\Xi}\end{array}$ & 离 & 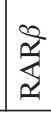 & $\sum$ & & ق气 & & & $\begin{array}{l}\mathbb{U} \\
\mathbb{J} \\
\end{array}$ & $\overrightarrow{\tilde{\theta}}$ & 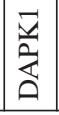 & 齐 & $\begin{array}{l}\tilde{x} \\
\underline{\Xi}\end{array}$ & 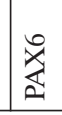 & $\frac{2}{2}$ & $\begin{array}{l}\vec{I} \\
\tilde{v} \\
\tilde{z} \\
\underline{\Sigma}\end{array}$ & $\sum_{k}^{E}$ \\
\hline
\end{tabular}


Table 4. Promoter hypermethylation of individual genes in NSCLC — univariate associations with clinicopathological features $\left(\mathrm{OR}^{\dagger}\right.$ and $\left.95 \% \mathrm{CI}\right)$

\begin{tabular}{|c|c|c|c|c|c|}
\hline Genes & Male vs. female & Age (per 10 years) & Smoking history & Pack-years ${ }^{1}$ & SSC vs. ADC \\
\hline CALCA & $0.73(0.28-1.90)$ & $0.94(0.58-1.52)$ & $1.43(0.57-3.57)$ & $0.88(0.51-1.51)$ & $2.21(0.89-5.47)$ \\
\hline $\mathrm{CDH} 1$ & $2.13(0.86-5.22)$ & $0.83(0.53-1.30)$ & $1.41(0.59-3.39)$ & $0.91(0.55-1.50)$ & $2.63(1.05-6.60)^{*}$ \\
\hline DAPK1 & $1.45(0.43-4.87)$ & $1.16(0.60-2.24)$ & $2.11(0.64-6.92)$ & $1.83(0.84-3.98)$ & $6.64(1.85-23.8)^{*}$ \\
\hline EVX2 & $0.94(0.38-2.33)$ & $1.12(0.70-1.80)$ & $1.16(0.47-2.85)$ & $0.82(0.48-1.41)$ & $2.16(0.89-5.29)$ \\
\hline IRX2 & $1.29(0.54-3.06)$ & $0.76(0.48-1.19)$ & $1.06(0.45-2.52)$ & $0.91(0.55-1.50)$ & $2.89(1.17-7.13)^{*}$ \\
\hline PAX6 & $1.04(0.37-2.88)$ & $1.19(0.70-2.03)$ & $0.78(0.27-2.25)$ & $0.93(0.51-1.69)$ & $1.04(0.37-2.88)$ \\
\hline $\operatorname{RAR} \beta$ & $0.75(0.31-1.81)$ & $1.13(0.72-1.77)$ & $0.50(0.20-1.23)$ & $0.60(0.36-1.01)$ & $0.92(0.39-2.19)$ \\
\hline RASSF1A & $0.75(0.28-2.05)$ & $0.98(0.59-1.62)$ & $0.58(0.20-1.62)$ & $0.73(0.41-1.30)$ & $0.31(0.09-0.99)^{*}$ \\
\hline TIMP3 & $1.09(0.46-2.60)$ & $1.09(0.70-1.71)$ & $1.09(0.46-2.60)$ & $1.27(0.76-2.11)$ & $2.51(1.01-6.21)^{*}$ \\
\hline Genes & Tumor size ${ }^{2}$ & Lymph node metastasis & Pleural indentation & Invasion or adhesion & Histologic stage $\mathrm{e}^{3}$ \\
\hline CALCA & $1.01(0.55-1.88)$ & $1.74(0.62-4.91)$ & $1.18(0.41-3.41)$ & $2.44(0.88-6.79)$ & $2.13(1.01-4.47)^{*}$ \\
\hline CDH1 & $1.20(0.67-2.13)$ & $1.02(0.41-2.51)$ & $1.79(0.67-4.76)$ & $0.96(0.41-2.27)$ & $1.11(0.62-2.02)$ \\
\hline DAPK1 & $1.81(0.72-4.54)$ & $5.17(0.64-42.0)$ & $1.63(0.33-8.02)$ & $1.70(0.43-6.67)$ & $1.24(0.50-3.08)$ \\
\hline EVX2 & $0.79(0.43-1.43)$ & $1.25(0.48-3.28)$ & $0.81(0.30-2.21)$ & $1.43(0.57-3.61)$ & $1.51(0.78-2.93)$ \\
\hline IRX2 & $1.57(0.88-2.83)$ & $1.17(0.47-2.88)$ & $1.37(0.52-3.63)$ & $0.86(0.36-2.01)$ & $1.14(0.63-2.07)$ \\
\hline PAX6 & $0.78(0.40-1.54)$ & $0.74(0.26-2.09)$ & $2.04(0.54-7.69)$ & $1.84(0.61-5.56)$ & $1.76(0.79-3.93)$ \\
\hline $\operatorname{RAR} \beta$ & $0.78(0.44-1.39)$ & $1.43(0.57-3.58)$ & $0.41(0.15-1.11)$ & $1.44(0.60-3.45)$ & $0.94(0.52-1.70)$ \\
\hline RASSF1A & $1.12(0.59-2.14)$ & $0.47(0.18-1.25)$ & $1.76(0.53-5.84)$ & $0.44(0.17-1.11)$ & $0.47(0.24-0.90)^{*}$ \\
\hline TIMP3 & $1.67(0.92-3.01)$ & $2.05(0.82-5.10)$ & $1.16(0.44-3.05)$ & $0.69(0.29-1.66)$ & $1.88(1.01-3.49)^{*}$ \\
\hline
\end{tabular}

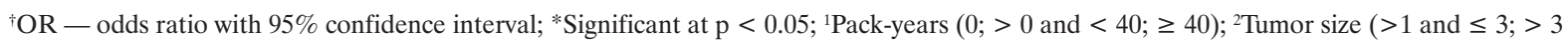
and $\leq 5 ;>5$ ); ${ }^{3}$ Histologic stage (I, II, III)

Table 5. Promoter hypermethylation of individual genes in NSCLC - multivariable models assessing age, pack-years, histologic type, and histologic stage $\left(\mathrm{OR}^{\dagger}\right.$ and $\left.95 \% \mathrm{CI}\right)$

\begin{tabular}{|c|c|c|c|c|}
\hline Genes & Age $^{1}$ & Pack-years $^{2}$ & SSC vs. ADC & Histologic stage $^{3}$ \\
\hline CALCA & $0.97(0.59-1.59)$ & $0.76(0.42-1.35)$ & $1.87(0.70-5.01)$ & $1.94(0.87-4.31)$ \\
\hline CDH1 & $0.81(0.51-1.29)$ & $0.81(0.47-1.39)$ & $3.14(1.14-8.65)^{*}$ & $0.87(0.45-1.65)$ \\
\hline DAPK1 & $1.10(0.55-2.20)$ & $1.53(0.67-3.48)$ & $8.07(1.75-37.2)^{*}$ & $0.60(0.20-1.80)$ \\
\hline EVX2 & $1.16(0.71-1.88)$ & $0.71(0.40-1.25)$ & $2.14(0.80-5.72)$ & $1.35(0.66-2.77)$ \\
\hline IRX2 & $0.74(0.46-1.18)$ & $0.80(0.47-1.38)$ & $3.54(1.29-9.73)^{*}$ & $0.86(0.44-1.65)$ \\
\hline PAX6 & $1.24(0.72-2.13)$ & $0.88(0.47-1.65)$ & $0.80(0.26-2.42)$ & $1.93(0.82-4.50)$ \\
\hline $\operatorname{RAR} \beta$ & $1.18(0.74-1.88)$ & $0.58(0.34-0.99)^{*}$ & $1.11(0.42-2.90)$ & $0.99(0.52-1.90)$ \\
\hline RASSF1A & $0.96(0.57-1.65)$ & $0.81(0.44-1.50)$ & $0.44(0.13-1.54)$ & $0.55(0.27-1.10)$ \\
\hline TIMP3 & $1.10(0.69-1.77)$ & $1.13(0.66-1.93)$ & $1.89(0.70-5.05)$ & $1.63(0.85-3.13)$ \\
\hline
\end{tabular}

${ }^{\dagger}$ OR — odds ratio with $95 \%$ confidence interval, adjusted for multiple comparisons; ${ }^{1}$ Age (per 10 years); ${ }^{2}$ Pack-years $(0 ;>0$ and $<40$; $\geq 40$ ); ${ }^{3}$ Histologic stage (I; II; III); *Significant at $\mathrm{p}<0.05$

duals. The results showed that the prevalence of concomitant methylation of five or more genes, six or more genes, seven or more genes, and eight or more genes in NSCLC were 65/96 (67.7\%), 48/96 (50\%), 37/96
(38.5\%), and 23/96 (24\%), respectively. In addition, there was a significantly increased frequency for concomitant hypermethylation of multiple genes in SCC compared to ADC (data not shown). 


\section{Discussion}

Epigenetic gene silencing is a molecular mechanism of silencing a gene by methylating its promoter region. Epigenetic silencing is involved in initiation and progression of human cancer [10], including NSCLC $[11,12]$. The aim of this study is to identify a panel of hypermethylated genes with high sensitivity and specificity for early detection of NSCLC and explore their associations with clinocopathologic features of cases using a Q-MSP approach. Hypermethylation of most of these genes, as a mechanism for their silencing, has been frequently reported in NSCLC [13-17]. Moreover, this study also involved three new methylation markers, EVX2, IRX2, and PAX6, which were highly specific for tumor-associated methylation, and little or no methylation was found in non-malignant lung tissue [18]. Our data showed that there were significant differences in the methylation level between cancerous and noncancerous lung tissues from six of nine genes, including CALCA, CDH1, EVX2, PAX6, $\operatorname{RAR} \beta$, and RASSF1A (Figure 1). It is worth noting that $100 \%$ diagnostic specificity, and excellent sensitivity, ranging from $44.9 \%$ to $84.1 \%$, was obtained for each of the nine genes (Figure 2), suggesting that these methylation markers play a role in tumor development and make them excellent candidates for early diagnosis and prognostic evaluation of NSCLC.

Our study found that promoter hypermethylation of most of the genes was strongly associated with histologic type. Among them, a number of genes were more frequently methylated in SCC than in ADC (Tables 3 and 4). Unlike what was observed in the present study, previous studies have indicated that a number of genes were more frequently methylated in ADC, not in SSC [17, 19, 20], which is new to the literature. Of note, similar to a previous study [21], the present study showed that hypermethylation of RASSF1A was negatively associated with smoking history, lymph node metastasis, invasion or adhesion. Importantly, RASSF1A was more frequently methylated in the early tumor stage (Table 4), suggesting that RASSF1A hypermethylation may be an early molecular event in the development of a lung tumor.

Our study did not find a significant association between hypermethylation of most of the genes and smoking history in NSCLC, except for CALCA gene. This accords with most studies [22-25], although tobacco smoking plays a central role in the development of lung cancer. However, several studies, as well as the present study, have reported aberrant methylation of some tumor-related genes was associated with tobacco smoking in lung cancer $[19,23,26]$, such as p16 and CALCA genes. It is possible that smoking-associated lung cancer is a complex disease which involves many unique genetic and epigenetic alterations. Thus, a better understanding of the molecular mechanisms underlying this disease would undoubtedly improve the clinical outcomes of patients with NSCLC.

A very recent study showed that a number of important tumor-related genes are more frequently methylated in females compared to males in NSCLC, suggesting that certain methylation events are associated with gender [17]. However, these differences have not been consistently found in most of the previous studies [20, 27, 28]. Similarly, hypermethylation of all of these genes was also not significantly associated with gender in the present study. It is possible that the differences in the promoter hypermethylation associated with gender may be related to geographical or cultural differences, and, more importantly, differences in environmental carcinogen exposure [29-31]. The discrepant results might also have been caused by genetic differences between the study populations [32]. Moreover, it is possible that these differences are attributable to chance as a result of the relatively small cohort of study subjects. Further study with more subjects will thus be needed.

Concomitant methylation of multiple genes is common, particularly in cancerous tissues, which is strongly associated with lung cancer risk $[33,34]$. In the present study, we identified a panel of the nine hypermethylated genes with excellent specificity and sensitivity, which were only methylated in cancerous tissues but not in noncancerous tissues. Among these nine genes, concomitant methylation of five or more genes was detected in $67.7 \%$ (65/96) of NSCLCs; methylation of eight or more genes was also detected in $24 \%$ (23/96) of NSCLCs, suggesting that methylation of these genes could be used as an indicator of a widespread methylation phenotype in NSCLC. Today, gene methylation in biological fluids is emerging as a biomarker that could be important for the early detection of lung cancer and monitoring prevention and intervention. Thus, our findings reinforce the use of this panel of hypermethylated genes as a biomarker for early detection and prognostic evaluation of lung cancer.

To sum up, we identified a panel of hypermethylated genes that differentiate cancerous tissues from noncancerous tissues, and demonstrated that promoter hypermethylation was strongly associated with histologic type. Importantly, highly frequent concomitant methylation of multiple genes was found in NSCLC, particularly in SCC, strongly suggesting that a methylated gene panel from sputum or plasma may have potential in noninvasively detecting NSCLC, as well as in evaluating the prognosis in post-operative NSCLC patients. 


\section{Acknowledgements}

This work was supported by the National Natural Science Foundation of China (No. 30901459 and 30973372) and the Fundamental Research Fund for the Central Universities.

\section{References}

1. Molina JR, Yang P, Cassivi SD, Schild SE, Adjei AA. Non-small cell lung cancer: epidemiology, risk factors, treatment, and survivorship. Mayo Clin Proc. 2008;83:584-594.

2. Jemal A, Siegel R, Ward E, Hao Y, Xu J, Thun MJ. Cancer statistics, 2009. CA Cancer J Clin. 2009;59:225-249.

3. Hoffman PC, Mauer AM, Vokes EE. Lung cancer. Lancet. 2000;355:479-85.

4. Baylin SB, Herman JG. DNA hypermethylation in tumorigenesis: epigenetics joins genetics. Trends Genet. 2000;16:168-174.

5. Jones PA , Baylin SB. The fundamental role of epigenetic events in cancer. Nat Rev Genet. 2002;3:415-428.

6. Tsou JA, Hagen JA, Carpenter CL, Laird-Offringa IA. DNA methylation analysis: a powerful new tool for lung cancer diagnosis. Oncogene. 2002;21:5450-5461.

7. Belinsky SA. Gene-promoter hypermethylation as a biomarker in lung cancer. Nat Rev Cancer. 2004;4:707-717.

8. Wu G, Mambo E, Guo Z et al. Uncommon mutation, but common amplifications, of the PIK3CA gene in thyroid tumors. J Clin Endocrinol Metab. 2005;90:4688-4693.

9. Hou P, Ji M, Xing M. Association of PTEN gene methylation with genetic alterations in the phosphatidylinositol3-kinase/AKT signaling pathway in thyroid tumors. Cancer. 2008;113:2440-2447.

10. Jones PA, Baylin SB. The epigenomics of cancer. Cell. 2007;128:683-682.

11. Herman JG, Baylin SB. Gene silencing in cancer in association with promoter hypermethylation. N Engl J Med. 2003; 349:2042-2054.

12. Baylin SB, Ohm JE. Epigenetic gene silencing in cancer a mechanism for early oncogenic pathway addiction? Nat Rev Cancer. 2006;6:107-116.

13. Zochbauer-Muller S, Fong KM, Virmani AK, Geradts J, Gazdar AF, Minna JD. Aberrant promoter methylation of multiple genes in non-small cell lung cancers. Cancer Res. 2001;61:249-255.

14. Topaloglu O, Hoque MO, Tokumaru Y et al. Detection of promoter hypermethylation of multiple genes in the tumor and bronchoalveolar lavage of patients with lung cancer. Clin Cancer Res. 2004;10:2284-2288.

15. Wang Y, Zhang D, Zheng W, Luo J, Bai Y, Lu Z. Multiple gene methylation of nonsmall cell lung cancers evaluated with 3-dimensional microarray. Cancer. 2008;112:1325-1336.

16. Brock MV, Hooker CM, Ota-Machida E et al. DNA methylation markers and early recurrence in stage I lung cancer. N Engl J Med. 2008;358:m1118-1128.

17. Hawes SE, Stern JE, Feng Q et al. DNA hypermethylation of tumors from non-small cell lung cancer (NSCLC) patients is associated with gender and histologic type. Lung Cancer. 2010;69:172-179.

18. Rauch TA, Zhong X, Wu X et al. High-resolution mapping of DNA hypermethylation and hypomethylation in lung cancer. Proc Natl Acad Sci USA. 2008;105:252-257.

19. Toyooka S, Maruyama R, Toyooka KO, et al. Smoke exposure, histologic type and geography-related differences in the methylation profiles of non-small cell lung cancer. Int J Cancer. 2003;103:153-160.
20. Gu J, Berman D, Lu C et al. Aberrant promoter methylation profile and association with survival in patients with non-small cell lung cancer. Clin Cancer Res. 2006;12:7329-7338.

21. Honorio S, Agathanggelou A, Schuermann M et al. Detection of RASSF1A aberrant promoter hypermethylation in sputum from chronic smokers and ductal carcinoma in situ from breast cancer patients. Oncogene. 2003;22:147-150.

22. Brabender J, Usadel H, Danenberg KD et al. Adenomatous polyposis coli gene promoter hypermethylation in non-small cell lung cancer is associated with survival. Oncogene. 2001;20:3528-3532.

23. Kim DH, Nelson HH, Wiencke JK et al. p16 (INK4a) and histology-specific methylation of $\mathrm{CpG}$ islands by exposure to tobacco smoke in non-small cell lung cancer. Cancer Res. 2001;61:3419-3424.

24. Kim DH, Nelson HH, Wiencke JK et al. Promoter methylation of DAP-kinase: association with advanced stage in non-small cell lung cancer. Oncogene. 2001;20:1765-1770.

25. Sanchez-Cespedes M, Decker PA, Doffek KM et al. Increased loss of chromosome 9p21 but not p16 inactivation in primary non-small cell lung cancer from smokers. Cancer Res. 2001; 61:2092-2096.

26. Kersting M, Friedl C, Kraus A, Behn M, Pankow W, Schuermann M. Differential frequencies of p16(INK4a) promoter hypermethylation, p53 mutation, and K-ras mutation in exfoliative material mark the development of lung cancer in symptomatic chronic smokers. J Clin Oncol. 2000;18:3221-3229 .

27. Nakata $\mathrm{S}$, Sugio $\mathrm{K}$, Uramoto $\mathrm{H}$ et al. The methylation status and protein expression of CDH1, p16(INK4A), and fragile histidine triad in nonsmall cell lung carcinoma: epigenetic silencing, clinical features, and prognostic significance. Cancer. 2006;106:2190-2199.

28. Vaissiere T, Hung RJ, Zaridze D et al. Quantitative analysis of DNA methylation profiles in lung cancer identifies aberrant DNA methylation of specific genes and its association with gender and cancer risk factors. Cancer Res. 2009; 69:243-242.

29. Issa JP, Baylin SB, Belinsky SA. Methylation of the estrogen receptor CpG Island in lung tumors is related to the specific type of carcinogen exposure. Cancer Res. 1996;56:3655-3658.

30. Moore LE, Huang WY, Chung J, Hayes RB. Epidemiologic considerations to assess altered DNA methylation from environmental exposures in cancer. Ann NY Acad Sci. 2003; 983:181-196.

31. Vuillemenot BR, Pulling LC, Palmisano WA, Hutt JA, Belinsky SA. Carcinogen exposure differentially modulates RAR-beta promoter hypermethylation, an early and frequent event in mouse lung carcinogenesis. Carcinogenesis. 2004;25:623-629.

32. Gilliland FD, Harms HJ, Crowell RE, Li YF, Willink R, Belinsky SA. Glutathione S-transferase P1 and NADPH quinone oxidoreductase polymorphisms are associated with aberrant promoter methylation of P16(INK4a) and $\mathrm{O}(6)$-methylguanine-DNA methyltransferase in sputum. Cancer Res. 2002;62:2248-2252.

33. Feng Q, Hawes SE, Stern JE et al. DNA methylation in tumor and matched normal tissues from non-small cell lung cancer patients. Cancer Epidemiol Biomarkers Prev. 2008; 17:645-654.

34. Belinsky SA, Schiller JH, Stidley CA. DNA methylation biomarkers to assess therapy and chemoprevention for non-small cell lung cancer. Nutr Rev. 2008;66 Suppl 1:S24-26. 